\title{
Vertex-transitive monocoronal tilings from isohedral tilings
}

Eduard Camangian Taganap ${ }^{1}$, Ma. Louise Antonette N. De Las Penas ${ }^{2}$

${ }^{1}$ Department Of Mathematics And Physics, Central Luzon State University, Science City Of Munoz, Philippines, ${ }^{2}$ Department of Mathematics, Ateneo de Manila University, Quezon City, Philippines E-mail: eduardtaganap@gmail.com

In this work, we present a class of Euclidean and hyperbolic tilings known as monocoronal tilings. A monocoronal tiling is where all its vertex-coronae are congruent. By a vertex-corona of a vertex of a tiling, we mean its vertex together with the tiles incident to it.

Vertex-transitive Euclidean monocoronal tilings are of importance in crystallography because as graphs they are considered to be uninodal "nets" and are useful in describing the structure of crystals [1, 2]. Moreover, studies on particular hyperbolic monocoronal tilings show projections of these tilings to three-periodic minimal surfaces in $\mathrm{E}^{3}$ to obtain crystalline three periodic nets [3]. In the hope of providing a wider range of tilings which could be used in describing and studying crystalline nets, we provide an approach of generating vertex-transitive monocoronal tilings.

In this presentation we discuss our methodology in constructing vertex-transitive monocoronal tilings from face-to-face isohedral tilings $\mathrm{T}$. The method requires considering the orbit $\mathrm{Hx}=\{\mathrm{hx}: \mathrm{h} \in \mathrm{H}\}$ of a point $\mathrm{x}$ (in the interior of tile $\mathrm{t}$ in $\mathrm{T}$ ) under a particular subgroup $\mathrm{H}$ of the symmetry group of $\mathrm{T}$. To form a monocoronal tiling, we connect by an edge two points in $\mathrm{Hx}$ that belong to adjacent tiles in $\mathrm{T}$. Such a method provides a systematic enumeration of vertex-transitive monocoronal tilings.

[1] Modern Methods of Crystal Structure Prediction (2011), edited by A. Organov, p. 1-28. Weinheim: Wiley-VCH Verlag \& Co. KGaA.

[2] Delgado-Friedrichs, O. et al. (2005). Journal of Solid State Chemistry 178, 2533-2554.

[3] Ramsden, S. et al. (2009). Acta Cryst. A 65, 81-108.

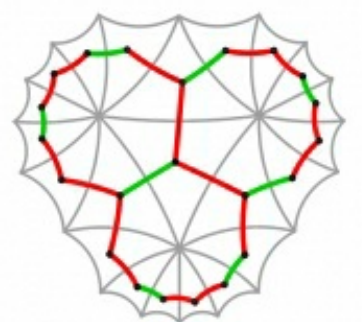

Vertex-corona of a monocoronal tiling from isohedral tiling $\left[9^{3}\right]$ where edges of the same color represent congruent edges.

Keywords: monocoronal tilings, hyperbolic tilings, crystalline nets 